\title{
Absorption of Succinate Diesters of Medium-Chain Fatty Alcohols Having Ability to Induce Nutritional Encephalomalacia in Starting Chicks
}

\author{
Haruhisa Ikumo and Minoru Yoshida \\ National Institute of Animal Industry, Chiba-shi \\ Received June 7, 1974
}

\begin{abstract}
Succinate diesters of medium-chain fatty alcohols $\left(\mathrm{C}_{6}, \mathrm{C}_{8}, \mathrm{C}_{10}, \mathrm{C}_{12}\right)$ was prepared to be studied on their ability to induce nutritional encephalomalacia in starting chicks and on the mechanism of their hydrolysis, absorption, and transport in chicks, using dilauryl succinate as positive control which possesses strong ability to induce encephalomalacia. It was revealed that all the succinate diesters used in this experiment, i.e., dilauryl succinate, monodecylmonolauryl succinate, monooctyl-monolauryl succinate, monohexyl-monolauryl succinate, didecyl succinate, dioctyl succinate, and dihexyl succinate had ability to induce encephalomalacia in starting chicks. It was observed that succinate diesters were hydrolysed into monoesters and free alcohols mainly in the region between jejunum and ileum, and absorbed to the portal vein in the form of monoester and free alcohol, not in intact form as diester, and transported to the liver. The possible proposal that monoesters will be most important compound for the induction of encephalomalacia is discussed.
\end{abstract}

In the series of studies to research for new dietary energy sources it was found that saturated higher alcohols $\left(\mathrm{C}_{10} \sim \mathrm{C}_{14}\right),{ }^{1)}$ aldehydes $\left(\mathrm{C}_{10}, \mathrm{C}_{12}\right),{ }^{17}$ glycol esters of medium-chain fatty acids, ${ }^{3}$ decyl acetate, ${ }^{11}$ lauryl stearate, ${ }^{12}$ and dilauryl succinate ${ }^{1,3)}$ possessed the ability of inducing nutritional encephalomalacia, one of typical vitamin E deficiency, in starting chicks. Fragility of the erythrocytes of the chicks fed these compounds was observed ${ }^{4 /}$ prior to the symptom of encephalomalacia, while exudative diathesis or muscular dystrophy have been never observed. Both encephalomalacia and fragility of the erythrocytes were completely prevented $^{4,5)}$ by the competitive dose of $\alpha$ tocopheryl acetate with the inducing compounds.

Of all the compounds which have been tested in this laboratory dilauryl succinate (DLS) is the strongest inducer of encephalomalacia, ${ }^{1)}$ and with 3 weeks of feeding period about $80 \%$ of the chicks fed the diet containing $12 \%$ of DLS show the symptom of encephalomalacia. ${ }^{11}$ In the earlier stage of the series of the studies, the ability of DLS to induce encephalomalacia was expected to be attributable completely to that of intramolecular laurylalcohol of DLS. It was revealed, however, that the ability was clearly inferior to that of DLS at the same dietary level. ${ }^{1 /}$ Therefore, the ability of DLS to induce encephalomalacia is not attributable solely to that of intramolecular laurylalcohol.

In the course of studyting the mechanism of the induction of encephalomalacia by DLS it will be most fundamental to investigate absorption and transport of DLS in chicks, although the digestibility of laurylalcohol and DLS in chicks were already reported to be $96 \%$ and $88 \%{ }^{\circ}{ }^{7)}$ respectively. It is the purpose of this paper to clarify whether in addition to DLS, other succinate diesters of medium-chain alcohols also have the ability to induce encephalomalacia in starting chicks, or not, and to study absorption and transport of these succinate diesters of medium-chain alcohols in chicks.

\section{MATERIALS AND METHODS}

Reagent. Dilauryl succinate (DLS) and monolauryl succinate (MLS) were kindly given from Kao Chemicals Ltd. $n$-Laurylalcohol, $n$-decylalcohol, $n$-octylalcohol, $n$ hexylalcohol, succinic anhydride, and dioctyl adipate 
were purchased from Wako Company Ltd. The purity of DLS, MLS, and medium-chain fatty alcohols were checked by gas chromatography, or infra red analysis, and it was revealed that the purity of the chemicals were more than $96 \%$ except $n$-decylalcohol which contained $20 \%$ of $n$-laurylalcohol as impurity, and that DLS contained neither MLS nor laurlalcohol as impurity, and MLS contained no laurylalcohol. $n$-Decylalcohol was used in the experiment after purified by twice fractional distillation, and checked its purity by gas chromatography.

Preparation of succinate diesters of medium-chain fatty alcohols. Succinate monoesters of alcohols, i.e., monocarboxylic acids, were obtained by heating the equivalent mixture of succinic anhydride and fatty alcohol at $120^{\circ} \mathrm{C}$ for $1 \mathrm{hr}$, and then succinate monoesters were further esterified to diesters with the other (or the same) alcohol at $50^{\circ} \sim 60^{\circ} \mathrm{C}$ by added adequate amount of $\mathrm{HCl}$. Seven kinds of diesters, i.e., monomethyl-monolauryl succinate (MMeMLS), monohexyl-monolauryl succinate (MHMLS), monooctylmonolauryl succinate (MOMLS), monodecyl-monolauryl succinate (MDMLS), dihexyl succinate (DHS), dioctyl succinate (DOS), and didecyl succinate (DDS) were thus obtained and used in the experiment.

Composition of diets. The composition of the basal diet was as follows, yellow corn: $60.0 \%$, soybean meal: $30.2 \%$, fish meal: $5.0 \%$, DL-methionine: $0.2 \%$, minerals: ${ }^{31} 4.1 \%$ (containing $0.1 \% \mathrm{Cr}_{2} \mathrm{O}_{3}$ ), and vitamins ${ }^{3)}$ without vitamin $\mathrm{E}: 0.5 \%$. The test and control diet were composed of $88 \%$ of the basal diet, and $12 \%$ of test compounds and corn starch, respectively, except that the test diet for MLS was composed of $7 \%$ of MLS and $93 \%$ of the basal diet.

Experimental procedure. Three experiment were carried out. In Expt. 1, absorption and transport of DLS and measurement of absorbabilty of DLS and laurylalcohol in chickens were studied. Meat type of cross bred male chickens (WCXWR) of about $1.5 \mathrm{~kg}$ of body weight were divided into 3 groups, and the diet containing $12 \%$ of DLS was given to 10 chickens, while the diet containing $12 \%$ of laurylalcohol and control diet were given to 5 chickens each, respectively. During one week of preliminary feeding period the chickens were accustomed to the diet, and fresh excreta from the fourth day to the fifth day in the preliminary period were collected from each chicken to measure digestibility of DLS and laurylalcohol. Excreta was homogenized with suitable amount of distilled water, and an aliquot of homogenate was taken to measure its contents of $\mathrm{Cr}_{2} \mathrm{O}_{3}$ spectrophotometrically at $370 \mathrm{~nm}$ by using phosphate reagent, ${ }^{81}$ while other aliquot of homogenate was used to determine the contents of DLS or laurylalcohol with gas chromatography. For gas chromatographic analysis an aliquot of homogenate was extracted overnight with ethylether after dehydration with $\mathrm{Na}_{2} \mathrm{SO}_{4}$, and the extract was methylated with diazomethane before gas chromatographic analysis. Determination of the contents of $\mathrm{Cr}_{2} \mathrm{O}_{3}$ and test compounds in the diet was also performed except no dehydration of the diet with $\mathrm{Na}_{2} \mathrm{SO}_{4}$.

Absorption and transport of DLS in chickens was studied as follows; chickens were starved $24 \mathrm{hr}$ after preliminary feeding of the diet containing $12 \%$ of DLS for one week, and then fed the DLS diet for $3 \mathrm{hr}$, and anethesized with intravenous injection of nembutal solution $(0.5 \mathrm{ml} / \mathrm{kg})$. Chickens were sacrificed to take heparinized blood samples from portal vein and jugular vein, and liver samples, and in addition, digestive canals from the proventriculus to the end of iluem. Digestive canals were divided into five regions, i.e., proventriculus + gizzard, duodenum, and three equal parts of length of jejunum + ileum. Extraction of the metabolites of DLS from plasma of portal vein and jugular vein and from the liver was carried out by employing Folch's method. On the other hand, boiling methanol, which has the ability to inactivate lipase activity, was used to extract the metabolites of DLS from digestive canal. After methylation with diazomethane, the extracts were analyzed with gas chromatography to detect the metabolites of DLS.

In Expt. 2, study on monolauryl succinate (MLS) was carried out. One hundred and fifty one-day-old male White Leghorn chicks were divided into 4 groups of 30 chicks each and 2 groups of 15 chicks each. A diet containing $7 \%$ of MLS, composed of $7 \%$ of MLS and $93 \%$ of basal diet, the diet containing $12 \%$ of DLS and $88 \%$ of basal diet, the diet containing $12 \%$ of laurylalcohol and $88 \%$ of basal diet, and the control diet were given to 30 chicks each. The diet containing $12 \%$ of MMeMLS, and the diet containing $7.4 \%$ of MLS $+4.6 \%$ of laurylalcohol, which correspond to the hydrolysates of $12 \%$ of DLS, were given to 15 chicks each. All the chicks were given the diet and water ad libitum for 5 weeks to investigate the ability of inducing encephalomalacia. After 5 weeks some of the survived chicks fed MLS $7 \%$ diet or MMeMLS $12 \%$ diet were sacrificed to clarify absorption and transport of MLS or MMeMLS in chicks with the same method as that in Expt. 1. Fresh excreta from the group of the chicks fed MLS diet was collected between the ninth and the tenth day of feeding period to measure the digestibility of MLS in chicks with the same method as that in Expt. 1.

In Expt. 3, succinate diesters of medium-chain fatty alcohols, namely MHMLS, MOMLS, MDMLS, DHS, DOS, and DDS were checked their ability to induce encephalomalacia and studied their absorption and transport on chicks. Eigthty one-day-old White Leghorn male chicks were divided into 8 groups of 10 chicks each. Each of the group was fed one of 8 kinds of diet containing $12 \%$ of 6 kinds of diesters 
mentioned above, and in addition, $12 \%$ of DLS as positive control and $12 \%$ of corn starch as negative control. Being investigated the symptom of encephalomalacia, chicks were fed the diet and water ad libitum for 22 days and at twenty-second day chicks surviving were sacrificed to study absorption and transport of test compounds in chicks. Fresh excreta from the group of the chicks fed the test diets were collected between the ninth and the tenth day of feeding period to measure the digestibility of test compounds in chicks with the same method as that in Expt. 1.

Digestibility. Digestibility $(\mathrm{D} \%$ ) was calculated as follows, $\mathrm{D}=\left(1-\frac{\mathrm{Se} \times \mathrm{Cd}}{\mathrm{Sd} \times \mathrm{Ce}}\right) \times 100$, where $\mathrm{Sd}$ represents the concentration of test compounds in the diet, and Se represents that of test compounds plus their intermetabolites in the excreta, while $\mathrm{Cd}$ and $\mathrm{Ce}$ represents the concentration of $\mathrm{Cr}_{2} \mathrm{O}_{3}$ in the diet and excreta, respectively. The methods for the measurement of the concentration of test compounds or $\mathrm{Cr}_{2} \mathrm{O}_{3}$ in the diets or excreta is described above.

Gas chromatography. All of the test compounds, i.e., medium-chain fatty alcohols $\left(\mathrm{C}_{6} \sim \mathrm{C}_{12}\right)$, succinate monoesters, succinate diesters, and palmitic acid were analyzed with gas chromatography by using temperature programming technique, and using $\mathrm{OV}-17$ as column packings. Succinate monoesters (possessing one carboxyl group) and palmitic acid were methylated with diazomethane prior to gas chromatographic analysis. Samples extracted with organic solvents in the experiments were all methylated before gas chromatographic analysis. Dioctyladipate was employed as internal standard for the determination of test compounds. In Fig. 1 is shown the standard gas chromatogram of test compounds.

\section{RESULT}

In every extract of five regions of the digestive canal of the chickens fed the diet containing $12 \%$ of DLS, there was enough detectable amount of MLS and laurylalcohol, which are hydrolysates of DLS, and DLS itself. In Table $I$ is shown the relative quantitative amount of these three compounds and palmitic acid as popular compound, taking the concentration of DLS as 100 . It was revealed that parts of DLS was hydrolyzed into MLS and laurylalcohol in digestive canal. The hydrolysis occurred at first in the stomach (proventriculus + gizzard) though the activity was very weak, and the strongest activity of the hydrolysis was observed in the small intestine. Detection of succinic acid was not tried since the column packings (OV-17) used in gas chromatography was hardly useful for detection of succinic acid.

In Table II is shown the result of analysis of the intermetabolites of DLS in the portal vein, liver, and jugular vein of the chickens fed the diet containing $12 \%$ of DLS, taking the concentration of MLS as 100 , where the amount of palmitic acid was measured to be compared

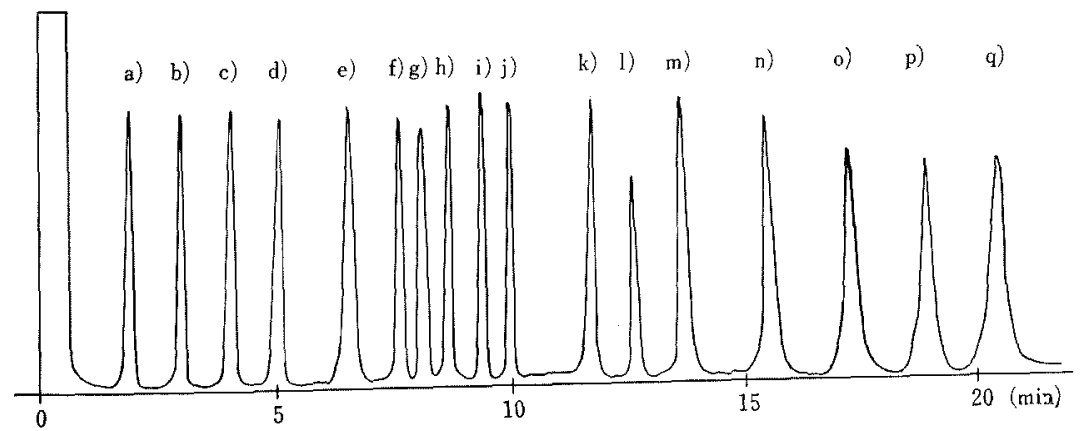

FIG. 1. Standard Gas Chromatogram* of Succinate Diesters, Succinate Monoesters, and Free Alcohols.

a) Hexylalcohol, b) Octylalcohol, c) Decylalcohol, d) Laurylalcohol, e) Monohexyl succinate, f) Monooctyl succinate, g) Palmitic acid, h) Monodecyl succinate, i) Dihexyl succinate, j) Monolauryl succinate, k) Dioctyl succinate, 1) Dioctyl adipate, ${ }^{* *}$ m) Monohexyl-monolauryl succinate, n) Monooctyl-monolauryl succinate, o) Didecyl succinate, p) Monodecyl-monolauryl succinate, q) Dilauryl succinate.

* Column Packings: OV-17, Temperature Programming: $80^{\circ} \sim 260^{\circ} \mathrm{C}, 10^{\circ} \mathrm{C} / \mathrm{min}$.

** Internal standard. 
Table I. Analysis of the Methanol Extract in the Digestive Canal of the Chick Fed DLS Diet

\begin{tabular}{lccccc}
\hline & $\begin{array}{c}\text { Proventriculus } \\
+ \\
\text { Gizzard }\end{array}$ & Duodenum & \multicolumn{3}{c}{ Jejunum+Ileum } \\
\cline { 4 - 6 } & $100^{a}$ & 100 & Upper & Middle & Lower \\
\hline Dilauryl succinate & $1.6 \pm 0.9$ & $6 \pm 2$ & $22 \pm 9$ & 100 & 100 \\
Monolauryl succinate & $2.2 \pm 1.3$ & $6 \pm 4$ & $17 \pm 10$ & $26 \pm 17$ & $18 \pm 14$ \\
Laurylalcohol & $8 \pm 5$ & $5 \pm 3$ & $12 \pm 4$ & $7 \pm 2$ & $33 \pm 20$ \\
Palmitic acid & & & & &
\end{tabular}

a) Peak area on gaschromatogram is shown by index taking peak area of dilauryl succinate as 100 .

Table II. Analysis of the Extract by the Folch's Method in the Portal Vein, Liver, AND Jugular VeIn of THE CHICKS FED DLS DIET

\begin{tabular}{lccc}
\hline & $\begin{array}{c}\text { Portal } \\
\text { vein }\end{array}$ & Liver & $\begin{array}{c}\text { Jugular } \\
\text { vein }\end{array}$ \\
\hline $\begin{array}{c}\text { Dilauryl } \\
\text { succinate }\end{array}$ & - $^{a}$ & - & - \\
$\begin{array}{l}\text { Monolauryl } \\
\text { succinate }\end{array}$ & $100^{b}$ & 100 & 100 \\
$\begin{array}{l}\text { Laurylalcohol } \\
\text { Palmitic acid }\end{array}$ & $55 \pm 30$ & $190 \pm 75$ & $28 \pm 10$ \\
\hline
\end{tabular}

a) Not detectable.

b) Peak area on gaschromatogram is shown by index taking peak area of monolauryl succinate as 100 .

with those of DLS, MLS and laurylalcohol since palmitic acid was normally enough detectable compound in chicks. Enough detectable amount of both MLS and laurylalcohol was observed in the portal vein, liver, and jugular vein of all the chicks fed the diet containing $12 \%$ of DLS, while there was no detectable amount of DLS itself. Average and standard deviation of digestibility of DLS was $69.3 \pm$ $10.1 \%$ for ten chickens and those of laurylalcohol $96.2 \pm 2.0 \%$ for five chickens, where absorbability of DLS was determined by measuring not only DLS but also MLS and laurylalcohol in the excreta of the chickens fed the diet containing $12 \%$ of DLS. In Table III is shown the result of feeding experiment for MLS for 5 weeks. In preliminary experiment, most of the chicks fed the diet containing $12 \%$ of MLS starved to death because of extremely poor appetite. Therefore, the diet containing $7 \%$ of MLS was used in Expt. 2. Receiving even low contents of $7 \%$ of MLS in the diet, however, chicks showed poor appetite to the diet and only one chick out of 30 chicks fed the diet containing $7 \%$ of MLS suffered encephalomalacia. On the other hand, the diet containing $12 \%$ of MMeMLS showed much better palatability to chicks, but no chicks suffered encephalomalacia. The mixed diet containing $7.4 \%$ of MLS and $4.6 \%$ of laurylalcohol which corresponded to the hydrolysates of $12 \%$ DLS showed the poorest palatability to chicks, and 4 chicks starved to death because of poor appetite, not due to encephalomalacia. $80 \sim 90 \%$ of all the chicks fed DLS as positive control suffered encephalomalacia, while, only one chick suffered encephalomalacia in the group fed the diet containing $12 \%$ of laurylalcohol.

In Tables IV and V, is shown the result of analysis of the extracts from the small intestine, portal vein, liver, and jugular vein of the chicks fed MLS and MMeMLS, respectively. As shown in Table IV, laurylalcohol was found as a hydrolysed product of MLS in the small intestine of the chicks fed the diet containing $7 \%$ of MLS, and its relative amount was about 18 and 38 of that of MLS in the upper and lower parts, respectively, taking the amount of MLS as 100. There was enough detectable amount of both MLS and laurylalcohol in the portal vein, liver, and jugular vein of the chicks fed MLS, and the relative amount of MLS was about 10 times as much as that of laurylalcohol. On the other hand, as shown in Table V, the amount of laurylalcohol in the small intestine of the chicks fed the diet containing $12 \%$ of 
Table III. The Result of Feeding Experiment for 5 Weeks on the Incidence of EnCePHalomalacia Using Monolauryl Succinate and MONOMETHYL-MONOLAURYL SUCCINATE

\begin{tabular}{lccccc}
\hline \multicolumn{1}{c}{$\begin{array}{c}\text { Diet } \\
(\%)\end{array}$} & $\begin{array}{c}\text { No. of } \\
\text { chicks }\end{array}$ & $\begin{array}{c}\text { Feed } \\
\text { intake }\end{array}$ & $\begin{array}{c}\text { Died/No. of } \\
\text { chicks }\end{array}$ & $\begin{array}{c}\text { Encephal- } \\
\text { omalacia }\end{array}$ & $\begin{array}{c}\text { Absorbability } \\
(\%)\end{array}$ \\
\hline Dilauryl succinate $(12 \%)$ & 30 & 62 & 24 & 26 & 96 \\
Monolauryl succinate $(7 \%)$ & 30 & 43 & 0 & 1 & \\
MMeMLS $^{(1)}(12 \%)$ & 15 & 72 & 0 & 1 \\
Laurylalcohol $(12 \%)$ & 30 & 56 & 0 & 0 \\
MLS + LA $^{(j)}$ & 15 & 31 & 4 & 0 \\
$(7.4 \%)(4.6 \%)$ & 30 & 100 & 0 & 0 \\
Control & & & & \\
\hline
\end{tabular}

a) Feed intake for 10 days from start is shown by index taking that of the control group as 100 .

b) Abbreviation of monomethyl-monolauryl succinate.

c) Abbreviation of monolauryl succinate.

d) Abbreviation of laurylalcohol.

Table IV. Analysis of the Extracts from the Small Intestine, Portal Vein, Liver, and Jugular Vein of 11 Chicks Fed Monolauryl Succinate

\begin{tabular}{lcccccc} 
& \multicolumn{2}{c}{ Small intestine } & & Portal V. & Liver & Jugular V. \\
\cline { 2 - 3 } & Upper & Lower & & & \\
\hline Monolauryl succinate & $100^{b}$ & 100 & & 100 & 100 & 100 \\
Laurylalcohol & $18 \pm 5$ & $38 \pm 11$ & & $7 \pm 4$ & $10 \pm 5$ & $13 \pm 14$ \\
\hline
\end{tabular}

a) Small intestine from duodenum to ileum was divided into two parts of equal length.

b) Peak area on gas chromatogram is shown by index taking peak area of monolauryl succinate as 100 .

Table V. Analysis of the Extracts from the Small Intestine, Portal Vein, Liver, and Jugular Vein of 5 Chicks Fed Monomethyl-monolauryl Succinate

\begin{tabular}{|c|c|c|c|c|c|}
\hline & \multicolumn{2}{|c|}{ Small intestine $\mathrm{e}^{a)}$} & \multirow{2}{*}{ Portal V. } & \multirow{2}{*}{ Liver } & \multirow{2}{*}{ Jugular V. } \\
\hline & Upper & Lower & & & \\
\hline Monolauryl succinate & $9 \pm 4$ & $5 \pm 3$ & $8 \pm 3$ & $-c)$ & $8 \pm 4$ \\
\hline Laurylalcohol & $100^{b 1}$ & 100 & 100 & $t+$ & 100 \\
\hline \multicolumn{6}{|l|}{ Monomethyl-monolauryl } \\
\hline succinate & $17 \pm 5$ & $12 \pm 4$ & - & - & 一 \\
\hline
\end{tabular}

a) Small intestine from duodenum to ileum was divided into two parts of equal length.

b) Peak area on gaschromatogram is shown by index taking peak area of laurylalcohol as 100 .

c) Not detectable.

MMeMLS was far superior to that of MMeMLS or MLS. There was no detectable MMeMLS in the portal vein, liver, or jugular vein of the chicks fed MMeMLS. Hydrolysed products from MMeMLS in the small intestine, i.e., laurylalcohol and MLS were detected both in the portal vein and jugular vein, where the amount of laurylalcohol was far more than that of MLS as observed in the small intestine.
Absorbability of MLS was $96 \%$ as shown in Table III.

Table VI presents the result of feeding experiment for succinate diesters of mediumchain alcohols. Dilauryl succinate as positive control induced encephalomalacia on 8 chicks out of 10 chicks fed DLS, while MDMLS, MOMLS, MHMLS, and DDS also induced encephalomalacia on 7,5,5, and 6 chicks out 
Table VI. The Result of Feeding Experiment for 22 Days for the InCidence of Encephalomalacia Induced by Succinate Diesters of Medium-Chain Fatty Alcohols

\begin{tabular}{|c|c|c|c|c|}
\hline $\begin{array}{l}\text { Diet } \\
(\%)\end{array}$ & $\begin{array}{l}\text { No. of } \\
\text { chicks }\end{array}$ & $\underset{\text { intake*1 }^{* 1}}{\text { Feed }}$ & Encephalomalacia & $\begin{array}{l}\text { Absorbability } \\
(\%)\end{array}$ \\
\hline $\begin{array}{r}\text { Dilauryl succinate } \\
(12 \%)\end{array}$ & 10 & 67 & $8^{a * 3}$ & 75 \\
\hline $\begin{array}{l}\text { Monodecyl-monolauryl succinate } \\
\qquad(12 \%)\end{array}$ & 10 & 65 & $7 \mathrm{ab}$ & 76 \\
\hline $\begin{array}{l}\text { Monooctyl-monolauryl succinate } \\
\qquad(12 \%)\end{array}$ & 10 & 64 & $5^{\mathrm{ab}}$ & 83 \\
\hline $\begin{array}{l}\text { Monohexyl-monolauryl succinate } \\
\qquad(12 \%)\end{array}$ & 10 & 64 & $5^{\mathrm{ab}}$ & 86 \\
\hline $\begin{array}{r}\text { Didecyl succinate } \\
(12 \%)\end{array}$ & 10 & 60 & $6^{a b}$ & 77 \\
\hline $\begin{array}{r}\text { Dioctyl succinate } \\
(12 \%)\end{array}$ & 10 & 56 & $O(1)^{* 2}$ & 90 \\
\hline $\begin{array}{r}\text { Dihexyl succinate } \\
(12 \%)\end{array}$ & 10 & 56 & $2 \mathrm{bc}$ & 90 \\
\hline Control & 10 & 100 & $0^{c}$ & - \\
\hline
\end{tabular}

*1 Feed intake for 10 days from start is shown by index taking that of control as 100 .

*2 One chick suffered encephalomalacia one day after experimental period.

*3 Difference between the incidence of encephalomalacia with a different sign in a block was significant statistically by $x^{2}$-test.

of 10 chicks each, respectively, fed these esters. Furthermore, DHS also induced encephalomalacia on 2 chicks out of 10 chicks. Dioctyl succinate (DOS) did not induce encephalomalacia during the experimental period of 22 days, but at twenty-third day after the termination of the experimental period one chick showed the symptom of encephalomalacia. In addition, specially poor appetite, or low digestibility was not observed among these esters, compared with those of DLS.

Table VII presents the result of analysis of succinate diesters and their intermetabolites in the small intestine, portal vein, liver, and jugular vein. In the small intestine succinate diesters were hydrolysed into their monoesters and free alcohols without exception, though parts of diesters still remained. There was enough detectable amount of monoesters and free alcohols in the protal vein, liver, and jugular vein, while all succinate diesters used in this experiment were undetectable, as observed in the chickens fed DLS diet in Expt.1. Quantitative difference was not recognized in the small intestine, portal vein, liver, or jugular vein between two kinds of monoesters produced from one diester, although the difference of hydrolytic affinity among fatty alcohol group could not be judged precisely from this experiment.

\section{DISCUSSION}

It has been reported that in mammals, fatsoluble compounds like triglycerides with longchain fatty acids are transported to the liver mainly via lymph vessels, while triglycerides with medium-chain fatty acids mainly via portal vein. ${ }^{9}$ On the other hand, it has been reported $^{10)}$ that in chicks, fat-soluble compounds are transported to the liver via portal vein since lymph vessels in chicks are far less developed compared with those in mammals, and that it was quite difficult to find lymph vessels in chicks with naked eyes. In the preliminary experiment, we were unable to find lymph vessels until they were coloured with black ink, and it was revealed that the thickness and the amount of fluid of lymph vessels were far much inferior to those of portal vein. Therefore, in this experiment portal vein was selected as fundamental route of test com- 


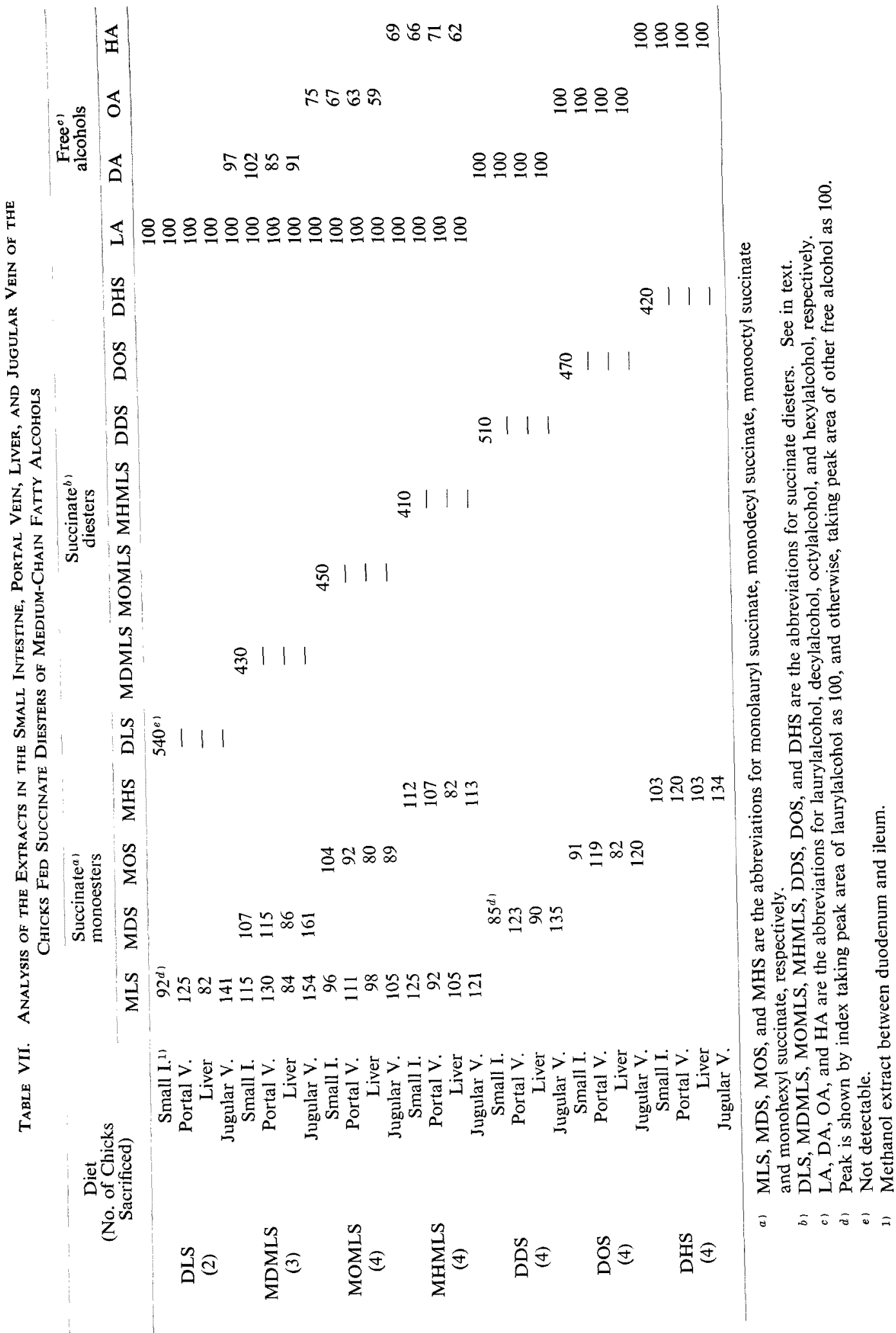


pounds to the liver. Jugular vein was regarded as the route of test compounds after their treatment in the liver.

The ability of DLS to induce encephalomalacia is not attributable solely to that of intramolecular laurylalcohol. This had been already reported in our previous paper, ${ }^{1 /}$ and was proved again in Expt. 2, as shown in Table III indicating the stronger inducing ability of DLS than that of laurylalcohol at the same dietary level, although the absorbability of laurylalcohol was superior to that of DLS. Though direct comparison of the ability of succinate diesters other than DLS and of the corresponding free alcohols was not carried out in this paper, the ability of the diesters was also suspected to be stronger than that of the latter, since the ability of decylalcohol was weaker than that of laurylalcohol. ${ }^{1)}$ According to the analytical technique employed in this experiment, absorption of DLS in intact form (unhydrolysed form) to the portal vein was not observed, although the possibility of DLS to be absorbed in intact form to the portal vein might be denied. There was enough detectable amount of both MLS and laurylalcohol in the portal vein, liver, and jugular vein without exception in the chicks fed DLS, and such experimental fact was also observed in regard to other succinate diesters of medium-chain alcohols, as shown in Table VII. In the pre-

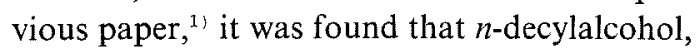
$n$-laurylalcohol, and $n$-myristylalcohol possessed the ability to induce encephalomalacia, and the order of the strength of the ability was laurylalcohol $>$ decylalcohol $>$ myristylalcohol. It was also revealed ${ }^{11}$ that neither $n$-hexylalcohol nor $n$-octylalcohol showed ability, one reason for which may be explained from their poor appetite and considerable toxicity to chicks. Succinate diesters of hexylalcohol and octylalcohol, i.e., dihexyl succinate and dioctyl succinate, both of which appeared to be less toxic than their free alcohols, however, showed the ability to induce encephalomalacia though they were rather weaker than that of DLS, MDMLS, MOMLS, MHMLS, and DDS, as shown in Table VI.
Succinate monoesters must be taken into account as an important intermetabolite of succinate diesters since it was revealed that monoesters were produced from their corresponding diesters in the small intestine, and transported to the liver via portal vein, and in addition, that diesters had the difficulty to be absorbed to the portal vein in intact form. In this report, only monolauryl succinate (MLS) was examined as succinate monoester. Although it was observed that the most part of MLS was absorbed to the portal vein in unhydrolyzed form as shown in Table IV, MLS, however, showed rather weak ability to induce encephalomalacia, which may be mainly attributable to very low appetite of MLS of the chick. On the other hand, monomethyl-monolauryl succinate (MMeMLS), which was prepared to improve the palatability to the chick, showed little ability to induce encephalomalacia. The reason may be explained from the experimental fact that laurylalcohol, the ability of which is rather weak as shown in Table III, was the major product from MMeMLS, and MLS was the minor product from MMeMLS as shown in Table $\mathrm{V}$, although monomethyl succinate produced together with MLS was not measured due to analytical difficulty.

The hypothesis will be suitable here that the model compound, i.e., $\mathrm{R}-\mathrm{O}-\stackrel{\mathrm{O}}{\mathrm{C}}-\mathrm{CH}_{2} \mathrm{CH}_{2}-$ $\mathrm{COOH}$ (succinate monoester of medium-chain fatty alcohols), will be more effective than R$\mathrm{OH}$ (medium-chain fatty alcohols) on the induction of encephalomalacia in starting chicks. Although the difference of hydrolytic affinity in the small intestine among intramolecular fatty alcohol group of succinate diesters can not be judged from this experiment, further hypothesis will be possible that $\mathrm{R}-\mathrm{O}-\stackrel{\mathrm{O}}{\mathrm{C}}-\mathrm{CH}_{2}-$ $\mathrm{CH}_{2}-\mathrm{COOH}$ may be more effective in the case of $\mathrm{R}: \mathrm{C}_{12}$ or $\mathrm{C}_{10}$ than that in the case of $\mathrm{R}: \mathrm{C}_{6}$ or $\mathrm{C}_{8}$ since the inducing effect of MHMLS, MOMLS, MDMLS, DLS, from which monolauryl succinate was produced in common, and of DDS, which produced only monodecyl succinate, was stronger than that of DHS and 
DOS which produced only monohexyl succinate and monooctyl succinate, respectively. It has been reported ${ }^{11}$ that the incidence of nutritional encephalomalacia in chicks is dependent on the amount of poly-unsaturated fatty acids in the diet. Kimura et $a l^{13}{ }^{13}$ reported that $\alpha$-tocopherol functioned as an anti-oxidant in the process of linoleic acid, i.e., in the reaction of the formation of 12-oxo-cis 9-octa-decenoic acid. An other role of the function of $\alpha$-tocopherol was studied by Lucy ${ }^{14}$ ) who proposed the physicochemical stabilization of erythrocytes membranes by vitamin $E$ that methyl group of the phytyl side-chain of $\alpha$-tocopherol interact with the cis double bonds of esterified arachidonic acid.

Double bond, however, is not present in the structure of either succinate diesters, or succinate monoesters, and in addition, the amount of peroxides unavoidably present in the diets used in this experiment may be very low as reported in the previous paper. ${ }^{3,5)}$ Therefore, the mechanism of the induction of encephalomalacia by succinate diesters appears to be different from what has been reported so far. Further study on succinate diesters and succinate monoesters will be performed hereafter to examine the hypotheses mentioned above, and to clarify the difference or similarity of the inducing mechanism to what has been reported.

\section{REFERENCES}

1) M. Yoshida and H. Hoshii, Agr. Biol. Chem., 35, 1610 (1971).

2) M. Yoshida, K. Kosaka and H. Hoshii, ibid., 37, 675 (1973).

3) M. Yoshida, H. Hoshii and H. Moromoto, ibid., 35, 822 (1971).

4) K. Kosaka and M. Yoshida, ibid., 37, 501 (1973).

5) M. Yoshida and H. Hoshii, ibid., 36, 755 (1972).

6) M. Yoshida, H. Morimoto and M. Matsui, ibid., 34, 1308 (1970).

7) M. Yoshida, H. Morimoto and R. Oda, ibid., 34, 1668 (1970).

8) M. Yoshida, K. Kosaka, S. Horii and S. Kameoka, Japan. Poultry Sci., 4, 24 (1967).

9) W. Stoffel, F. Chu and E. H. Ahrens, Jr., Anal. Chem., 31, 307 (1959).

10) J. H. Wiley and G. A. Leveille, J. Nutr., 103, 829 (1973).

11) A. Bensadoun and A. Rothfeld, Proc. Soc. Exp. Biol. Med., 141, 814 (1972).

12) J. Green and J. Bunyan, Nutr. Abtr. Rev., 39, 321, (1969).

13) H. Kimura and F, A. Kummerow, Arch. Biochem. Biophs., 102, 86 (1963).

14) J. A. Lucy, Ann. N. Y. Acad., Sci, , 203, 4 (1972). 